\title{
Reduced pelvic pain in women with endometriosis: efficacy of long-term dienogest treatment
}

\author{
Felice Petraglia • Daniela Hornung $\cdot$ Christian Seitz $\cdot$ \\ Thomas Faustmann · Christoph Gerlinger · \\ Stefano Luisi · Lucia Lazzeri · Thomas Strowitzki
}

Received: 28 March 2011 / Accepted: 30 May 2011 / Published online: 17 June 2011

(c) The Author(s) 2011. This article is published with open access at Springerlink.com

\begin{abstract}
Purpose To investigate the efficacy and safety of dienogest as a long-term treatment in endometriosis, with follow-up after treatment discontinuation. The study included women with endometriosis, who had previously completed a 12-week, placebo-controlled study of dienogest, who participated in an open-label extension study for up to 53 weeks. Thereafter,
\end{abstract}

Data from this study have been presented, in part, at the XIX FIGO World Congress of Gynecology \& Obstetrics, Cape Town, South Africa, October 4-9, 2009, and the American Society for Reproductive Medicine 65th Annual Meeting, Atlanta, GA, USA, October 17-21, 2009.

F. Petraglia $(\varangle) \cdot$ S. Luisi $\cdot$ L. Lazzeri

Department of Pediatrics, Obstetrics and Reproductive Medicine,

University of Siena, Viale R. Bracci N. 16, 53100 Siena, Italy

e-mail: petraglia@unisi.it

D. Hornung

Department of Obstetrics and Gynecology,

University of Schleswig-Holstein, Campus Lübeck,

Lübeck, Germany

C. Seitz

Global Clinical Development Women's Healthcare,

Bayer HealthCare Pharmaceuticals, Berlin, Germany

T. Faustmann

Global Medical Affairs Women's Healthcare,

Bayer HealthCare Pharmaceuticals, Berlin, Germany

C. Gerlinger

Global Biostatistics, Bayer HealthCare Pharmaceuticals,

Berlin, Germany

T. Strowitzki

Department of Gynecological Endocrinology and Reproductive

Medicine, University of Heidelberg, Heidelberg, Germany a patient subgroup was evaluated in a 24 -week follow-up after treatment discontinuation.

Methods A multicenter study performed in Germany, Italy and Ukraine. Women with endometriosis were enrolled at completion of the placebo-controlled study $(n=168)$. All women received dienogest ( $2 \mathrm{mg}$ once daily, orally) and changes in pelvic pain (on a visual analog scale), bleeding pattern, adverse events and laboratory parameters were evaluated during and after treatment.

Results The completion rate among women who entered the open-label extension study was $90.5 \%(n=152)$. A significant decrease in pelvic pain was shown during continued dienogest treatment $(P<0.001)$. The mean frequency and intensity of bleeding progressively decreased. Adverse events, rated generally mild or moderate, led to withdrawal in four patients $(2.4 \%)$. No clinically relevant changes in laboratory parameters were observed. During treatmentfree follow-up $(n=34)$, the reduction in pelvic pain persisted, while bleeding frequency and intensity returned to normal patterns.

Conclusions Long-term dienogest showed a favorable efficacy and safety profile, with progressive decreases in pain and bleeding irregularities during continued treatment; the decrease of pelvic pain persisted for at least 24 weeks after treatment cessation.

Keywords Dienogest · Endometriosis · Progestins · Pain · Safety

\section{Introduction}

Endometriosis is a chronic, benign disease causing symptoms of pelvic pain, dysmenorrhea and dyspareunia that often relapse after surgical therapy [1]. The aim of most 
medical therapies is to decrease the symptoms in order to improve quality of life, but many are associated with adverse effects that restrict their long-term use.

Gonadotropin-releasing hormone $(\mathrm{GnRH})$ agonists are associated with symptoms of estrogen deprivation (e.g., hot flushes, vaginal dryness, headache and decreased libido) and with bone demineralization that restricts use to 6 months in the absence of add-back therapy [2,3]. In addition, the duration of symptom relief following treatment cessation is typically short, decreasing the cost-effectiveness of $\mathrm{GnRH}$ agonists as compared to other approaches [2].

Combined oral contraceptives, while widely used to manage symptoms of endometriosis, are not approved for this indication in most countries and their use is largely unsupported by solid clinical trial evidence [4]. One study has demonstrated that the use of a low-dose oral contraceptive pill is an effective treatment for dysmenorrhea associated with endometriosis [5].

The widespread use of danazol in endometriosis is limited by adverse effects typical of androgenic steroids, such as alterations in lipid profile, weight gain, edema, acne, hirsutism and oily skin [6], although, in deeply infiltrating endometriosis, vaginal use may increase compliance [7].

Progestins are a recommended treatment for the pain associated with endometriosis, but a number of agents in this class are associated with androgenic effects and weight gain at the doses that are required for efficacy [8]. Longterm use of depot medroxyprogesterone acetate preparations impacts adversely on bone mineral density (BMD) [9]. Norethisterone acetate is a potent progestin derivative of 19-norethisterone and is valid for use in patients with endometriosis [8]; however, its main side effects include breast tenderness, weight gain, acne and hirsutism, in addition to breakthrough bleeding. Levonorgestrel (LNG) has also been used for treating women with symptomatic minimal-to-moderate endometriosis [10], leading to improvements in the severity and frequency of pain and menstrual symptoms. The LNG-intrauterine device (LNG-IUD) is an effective treatment for rectovaginal endometriosis in women who have previously undergone conservative surgery without excision of deep lesions [11]. However, the LNG-IUD is not approved for the treatment of endometriosis.

There remains, therefore, a need for an effective, safe and well-tolerated medical therapy that can be used in the long-term management of endometriosis. Dienogest is a selective progestin that combines the pharmacologic properties of 19-norprogestins and progesterone derivatives, offering a potent progestogenic effect at the endometrium. Clinical trials of 12-24 weeks' duration have shown that dienogest (at a dose of $2 \mathrm{mg} /$ day) provides effective pain relief equivalent to GnRH agonists, a reduction of endometriotic lesions and a favorable safety and tolerability profile in endometriosis [12-14].
A recent Japanese study investigated the efficacy of 52 weeks of dienogest administration for the treatment of endometriosis [15] and reported a marked or moderate improvement in symptoms in $90.6 \%$ of patients, with low rates of adverse event-related discontinuations and little impact on BMD.

In a recent, double-blind, placebo-controlled European study of 198 women with endometriosis, dienogest for 12 weeks was significantly more effective than placebo in reducing endometriosis-associated pelvic pain (EAPP), with low rates of treatment-related adverse events [16]. With the aim to investigate the long-term safety and efficacy of continuous dienogest treatment of endometriosis in this European network, a 1-year, open-label extension study to this placebo-controlled study was performed. In addition, at completion of the extension study, a subgroup of women was followed for 24 weeks without dienogest treatment.

\section{Materials and methods}

Women with laparoscopically confirmed endometriosis who participated in a 12-week, multicenter, randomized, double-blind, placebo-controlled study of dienogest treatment at $2 \mathrm{mg}$ once daily [16] were offered and accepted participation in an open-label, single-arm extension study of dienogest treatment $(n=168)$. The extension study was conducted at 28 centers in Germany $(n=17)$, Italy $(n=5)$ and Ukraine $(n=6)$ between July 2004 and November 2007. The duration of treatment was initially planned to be 36 weeks, but was extended to 52 weeks during the course of the study. Twenty-six patients completed the study before this prolongation was introduced to the protocol. For the final visit, a deviation of +1 week was allowed, resulting in a maximum treatment duration of 12 weeks (in the placebo-controlled study) plus 53 weeks in the current study (total 65 weeks). An additional 24 weeks, treatmentfree follow-up was conducted in a patient subgroup $(n=34)$ at six study centers on completion of the extension study. Therefore, an overall total of 90 weeks of study was conducted.

The study protocol was approved by local independent ethics committees and all participants provided written, informed consent before study enrolment. The study was conducted in accordance with the amended version of the Declaration of Helsinki and complied with Good Clinical Practice guidelines.

Inclusion and exclusion criteria for participation in the placebo-controlled study are described in detail elsewhere [16]. In brief, the inclusion criteria for women aged 18-45 years included histologically proven endometriosis (stages 1-4) at laparoscopy $\leq 12$ months before study entry [17] and an 
EAPP score of at least $30 \mathrm{~mm}$ on a $100-\mathrm{mm}$ visual analog scale (VAS) at screening and baseline visits [16]. All women, who completed their allocated treatment with either dienogest or placebo in the placebo-controlled study, without major protocol violations, were eligible for inclusion in the extension study. All patients at specific study centers who successfully completed the extension study were subsequently eligible for inclusion in the follow-up study.

Dienogest was administered at a dose of $2 \mathrm{mg}$ once daily, orally, for up to 53 weeks to all women during the extension study. The first dienogest tablet during the extension study was taken on the day after medication ended in the placebo-controlled study. Women completed a daily diary card throughout the extension study to monitor treatment compliance. Investigators recorded the use of any concomitant medications.

The main study variables were (1) EAPP, which was assessed by VAS score every 4 weeks to summarize the previous 4-week period (where $0 \mathrm{~mm}$ represents absence of pain and $100 \mathrm{~mm}$ indicates unbearable pain); (2) uterine bleeding pattern, which was assessed over 90-day periods according to the method of Gerlinger et al. [18]. Women documented the presence and intensity of bleeding on daily diary cards, from which the frequency and duration of bleeding events were calculated.

Patient visits were planned at baseline and at weeks 2, $13,25,36$ and 52 of the extension study, as well as at weeks 12 and 24 during the treatment-free follow-up.

Adverse events were documented at all study visits and coded according to the Medical Dictionary for Regulatory Activities Primary System Organ Class. Adverse events were rated treatment-related at the discretion of the investigator. Laboratory assessments included hematology, blood chemistry, liver enzyme, lipid and estradiol concentration measurements (Laboratorium für Klinische Forschung, Kiel, Germany) and dipstick urinalysis ("Combur-9"; Roche, Germany). Gynecologic examination (including cervical cytological smears), breast examination, vital sign assessments (blood pressure and heart rate) and body weight measurements were performed at predefined study visits. Abnormal findings at gynecologic and breast examination were reported as adverse events. Urine pregnancy testing was performed at home every 4 weeks and at study visits.

Quality of life assessments by the Short Form 36 Health Survey Questionnaire $\left(\mathrm{SF}-36^{\circledR}\right)$ were performed at the baseline and the end of the follow-up study to measure changes in mental and physical components.

\section{Statistical analyses}

Analyses are based on the full analysis set (FAS), which included all women who took at least one dose of study medication and provided at least one observation after dosing. Subgroup analyses are reported for women treated with dienogest or placebo during the previous placebocontrolled study ("prior-dienogest" and "prior-placebo" groups, respectively).

The efficacy and safety analyses in the extension and follow-up studies are presented primarily as descriptive statistics. The null hypothesis of whether the change in EAPP from randomization in the placebo-controlled study to the end of the extension study was equal to 0 was exploratively tested using the one-sample $t$ test.

The sample size of the extension study was determined by the number of women who were eligible and willing to participate at completion of the placebo-controlled study. According to the guidelines from the International Conference on Harmonization, the extent of population exposure to assess clinical safety requires at least 100 patients studied over 1 year. Assuming a dropout rate of $25 \%$, the extension study, therefore, required inclusion of a minimum of 133 women.

\section{Results}

Among women who completed the placebo-controlled study ( $n=188), 168$ entered the extension study and were included in the FAS (Fig. 1). A total of 152 women (90.5\%) completed the planned duration of the extension study ( $n=17,36$ weeks; $n=135,52$ weeks). No predominant reason for withdrawal was identified among women who discontinued. Treatment compliance, measured by daily diary cards during the extension study, was $98.3 \%$. A subgroup of 34 women at six study centers began the 24 weeks, treatment-free follow-up, with completion by 31 (91.2\%) (Fig. 1).

\section{Pelvic pain}

The mean VAS score at baseline of the extension study was $34.08 \mathrm{~mm}$ (standard deviation $[\mathrm{SD}] \pm 21.60 \mathrm{~mm}$ ) in the total population, resulting from mean scores of $27.89( \pm 20.24) \mathrm{mm}$ in the dienogest-treated and $40.73( \pm 21.14) \mathrm{mm}$ in the placebo-treated subgroup. The mean VAS score progressively and significantly decreased to $11.52( \pm 11.26) \mathrm{mm}$ at the end of the extension study in the total population $(9.72 \pm 7.44 \mathrm{~mm}$ in the prior-dienogest group and $13.49 \pm 14.14 \mathrm{~mm}$ in the prior-placebo group) (Fig. 2). The mean VAS score was statistically significantly reduced by $43.2( \pm 21.7) \mathrm{mm}$ over the total treatment period of 65 weeks (i.e., the placebo-controlled plus extension study; $P<0.001)$.

During the treatment-free follow-up, the mean VAS score increased slightly from the end of the extension study to 16.29 $( \pm 14.08) \mathrm{mm}$ at week 12 and $14.56( \pm 9.55) \mathrm{mm}$ at week 24. 
Fig. 1 Patient disposition during the extension study and the treatment-free follow-up period

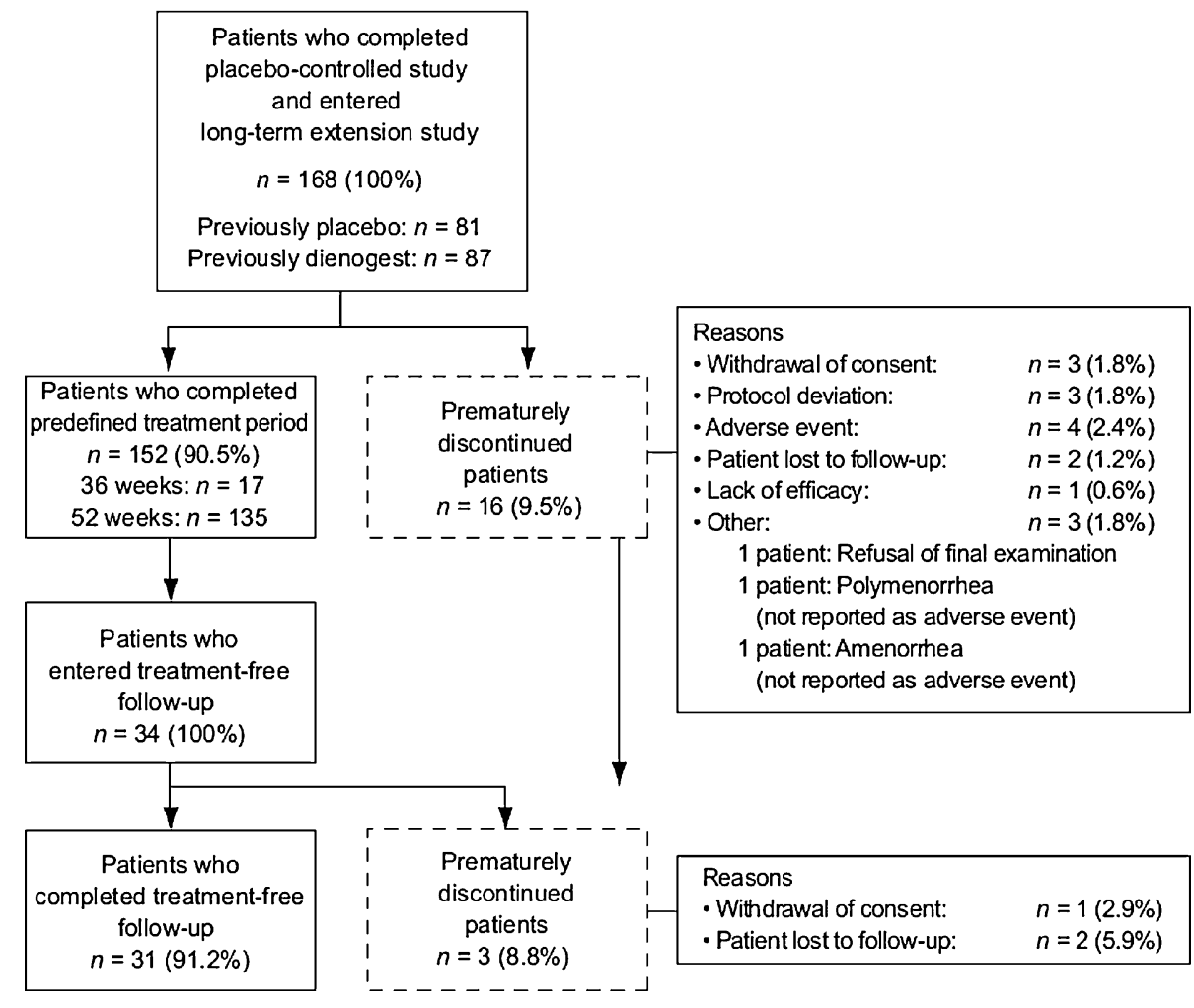

Subgroup analysis during the extension study showed that both the prior-dienogest and the prior-placebo subgroups of the placebo-controlled study experienced decreases in the number of bleeding/spotting days, number of spotting episodes and duration of bleeding/spotting episodes. These decreases were greater in the prior-placebo than in the prior-dienogest subgroup.

When comparing the frequency of maximal intensity of bleeding between 90-day reference periods 1 and 4, a substantial increase in the "no bleeding" category, i.e., women who never bled during the entire reference period (from 5.5 to $23.5 \%$ of women) and a decrease in the "heavy bleeding" category (from 9.8 to $2.2 \%$ ) was shown (Fig. 3). During treatment-free follow-up, bleeding returned to normal cyclic patterns and intensity within 4-6 weeks. No patients reported amenorrhea or spotting during the final 90-day reference period of treatment-free follow-up.

\section{Safety variables}

Laboratory parameters (Table 1), vital signs and body weight remained stable or underwent minimal changes during the extension study. In patients whose body weight was assessed at both baseline and after 52 weeks of treatment $(n=124)$, a mean change of $0.58( \pm 2.09) \mathrm{kg}$ was recorded. Laboratory parameters continued to show no or minimal changes during treatment-free follow-up, while vital sign and body weight assessments provided stable results. 


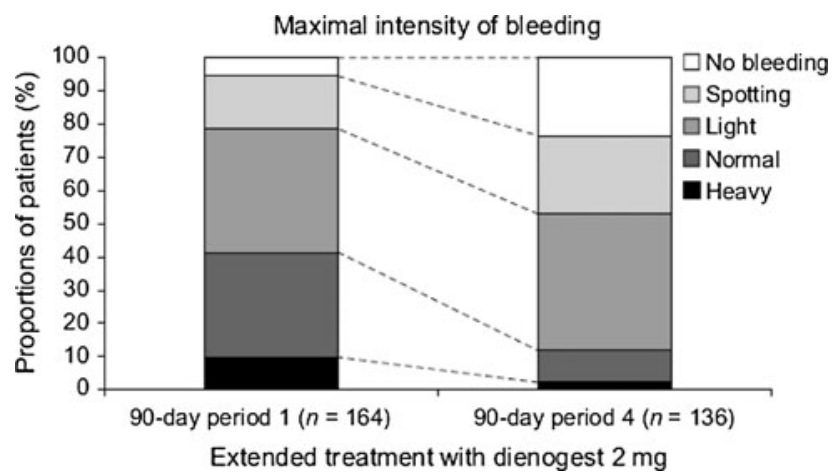

Fig. 3 Frequencies of maximal intensity of bleeding in 90-day reference periods 1 and 4 during dienogest treatment in the extension study (FAS) FAS full analysis set

Adverse events considered potentially drug related by the investigators developed in 27/168 women (16.1\%) during the extension study, including breast discomfort $(n=7 ; 4.2 \%)$, nausea $(n=5 ; 3.0 \%)$ and irritability $(n=4$; $2.4 \%)$. The maximal intensity of treatment-related adverse events was rated mild or moderate in the majority $(92.5 \%)$ of cases. Three women (1.8\%) experienced one serious adverse event each of cholelithiasis, chronic sinusitis and depression; of these, only the case of depression, which developed after approximately 4 months of study drug treatment, was considered to be possibly treatment related. Four women $(2.4 \%)$ experienced four adverse events (i.e., weight increase, migraine, depression and breast pain) that led to premature study discontinuation (Fig. 1). Two women $(1.2 \%)$ discontinued the study due to bleedingrelated events (amenorrhea and polymenorrhea), which were not recorded as adverse events. Abnormal cervical smear findings were recorded in two women, which were considered unlikely to be treatment related.

During treatment-free follow-up, 7/34 women (20.6\%) developed de novo adverse events, including headache in two women (5.9\%). One woman developed an ovarian cyst at the end of the follow-up period that was considered to be unrelated to previous study drug intake. Another woman had a successful, planned pregnancy, with conception estimated at approximately 6 months after ceasing treatment. Quality of life assessment by the SF- $36^{\circledR}$ indicated minimal change in mental or physical sum scores during the followup period.

Table 1 Selected laboratory parameters during dienogest treatment in the extension study

\begin{tabular}{|c|c|c|c|c|c|}
\hline \multirow[t]{2}{*}{ Parameter } & \multicolumn{2}{|c|}{$\begin{array}{l}\text { Baseline of extension } \\
\text { study }\end{array}$} & \multicolumn{2}{|c|}{$\begin{array}{l}\text { Absolute change from } \\
\text { baseline to end } \\
\text { of treatmenta }\end{array}$} & \multirow[t]{2}{*}{ Normal range } \\
\hline & $n$ & Mean \pm SD & $n$ & Mean \pm SD & \\
\hline \multicolumn{6}{|l|}{ Hematology } \\
\hline Erythrocytes $\left(10^{12} / \mathrm{L}\right)$ & 167 & $4.46 \pm 0.34$ & 155 & $-0.01 \pm 0.30$ & $3.6-5.0$ \\
\hline Leukocytes $\left(10^{9} / \mathrm{L}\right)$ & 166 & $5.94 \pm 1.82$ & 154 & $0.21 \pm 2.15$ & $4.0-10.0$ \\
\hline Platelets $(\mathrm{g} / \mathrm{L})$ & 167 & $248.4 \pm 66.40$ & 155 & $-1.3 \pm 60.60$ & $150-450$ \\
\hline $\mathrm{HbA}_{1 \mathrm{c}}(\%)$ & 168 & $5.16 \pm 0.31$ & 157 & $-0.13 \pm 0.29$ & $4.3-6.1$ \\
\hline \multicolumn{6}{|l|}{ Serum lipid concentrations } \\
\hline Total cholesterol (mmol/L) & 168 & $4.86 \pm 0.97$ & 161 & $-0.05 \pm 0.94$ & $4.14-6.73$ \\
\hline HDL cholesterol (mmol/L) & 168 & $1.53 \pm 0.33$ & 161 & $-0.04 \pm 0.36$ & $1.09-2.28$ \\
\hline LDL cholesterol (mmol/L) & 168 & $2.91 \pm 0.75$ & 161 & $0.10 \pm 0.71$ & $1.97-5.65$ \\
\hline Triglycerides (mmol/L) & 168 & $1.05 \pm 0.61$ & 161 & $0.03 \pm 0.57$ & $0.80-1.94$ \\
\hline \multicolumn{6}{|l|}{ Liver enzymes } \\
\hline Alkaline phosphatase (U/L) & 168 & $54.2 \pm 17.61$ & 161 & $3.6 \pm 17.36$ & $35-104$ \\
\hline Gamma glutamyl transferase (U/L) & 168 & $18.1 \pm 16.40$ & 161 & $2.3 \pm 23.66$ & $5-39$ \\
\hline Alanine aminotransferase (U/L) & 168 & $15.2 \pm 11.35$ & 161 & $1.6 \pm 13.61$ & $0-31$ \\
\hline Cholinesterase (U/L) & 168 & $7,463.4 \pm 1,421.20$ & 161 & $-116.9 \pm 1,473.26$ & $3,650-11,250$ \\
\hline \multicolumn{6}{|l|}{ Hormones } \\
\hline Estradiol (nmol/L) & 163 & $0.26 \pm 0.32$ & 151 & $0.02 \pm 0.44$ & $0.03-1.03$ \\
\hline
\end{tabular}

Values are number of women or mean \pm SD

$H b A_{l C}$ hemoglobin $\mathrm{A}_{1 \mathrm{C}}, H D L$ high-density lipoprotein, $L D L$ low-density lipoprotein

${ }^{\text {a }}$ Includes patients with a predefined treatment duration of 36 or 52 weeks, respectively 


\section{Discussion}

The present study shows that dienogest treatment for up to 65 weeks is associated with reduced pelvic pain in women with endometriosis, and that this effect persists for at least 24 weeks after the end of treatment. Previous studies of dienogest treatment for periods of 12-24 weeks reported superior efficacy relative to placebo [16] and equivalent efficacy compared with leuprolide acetate and buserelin acetate $[14,19]$ in relieving the symptoms of endometriosis. The present findings suggest that dienogest represents an effective treatment over the long term. The observation that pain relief is also largely maintained during the 24 weeks after treatment cessation may indicate that dienogest—at least for a certain period—sustains a beneficial effect that exceeds the period of symptomatic treatment. One plausible explanation for this effect is the reduction in endometriotic lesions observed during dienogest treatment [13], although additional explanations for the sustained reduction in pain are possible. Dienogest and other progestins have a number of putative mechanisms of action in endometriosis, including reduced growth, proliferation and neoangiogenesis of lesions [20,21] as well as an antiinflammatory effect [22].

Assessment of uterine bleeding by a number of analyses showed that dienogest is associated with a desynchronized uterine bleeding in the short term, but with a progressive reduction in the frequency and intensity of bleeding during continued treatment. Irregular bleeding has also been reported in short-term studies of dienogest treatment $[13,16,19]$. The bleeding pattern associated with dienogest appeared to be generally acceptable, as only 2 of 168 women in the extension study discontinued treatment due to changes in their bleeding pattern. The incidence of menorrhagia was lower in this study than in the Japanese long-term study, which reported a $71.9 \%$ incidence of menorrhagia; in that study, 18 of 135 patients (13.3\%) discontinued treatment for various reasons [15].

Irregular uterine bleeding is a known common adverse effect of all long-term progestin treatments [8]. Informing women of the bleeding profile of dienogest before they initiate treatment will likely enhance their long-term adherence to therapy in light of the accompanying improvement in painful symptoms.

The safety variables included in the extension study also indicated an acceptable profile for dienogest. The lack of changes in laboratory parameters supports previous trials that investigated the metabolic effects of dienogest $[13,14$, $16,23]$. In addition, there were minimal changes in mean body weight, consistent with the previous dienogest experience $[13,14,16]$.

This study contributes to the evidence base on medications investigated for the long-term treatment of endometri- osis. The strengths of the current study include the assessment of clinically relevant outcomes, the long-term (1 year) treatment duration, and a treatment-free follow-up, which are pertinent for the management of patients with endometriosis. The data from the follow-up period may be especially relevant for patients who wish to become pregnant after a course of medical therapy. Potential weaknesses of the study are its open-label design and the recruitment of patients who had participated in an earlier placebo-controlled study, which included a proportion who responded favorably to prior dienogest therapy. The low and comparable discontinuation rates in both subgroups, during and at the end of the placebo-controlled study indicate that this theoretical concern did not introduce a major bias.

In summary, this large-scale, long-term study showed that dienogest treatment induces a sustained reduction in EAPP associated with a favorable safety and tolerability profile. The observation of prolonged pain relief even after the return of normal menses suggests a sustained effect on endometrial lesions. Bleeding irregularities demonstrated a tendency toward reduced frequency and intensity during continued treatment. Therefore, dienogest may represent an effective long-term treatment option for women with endometriosis.

Acknowledgments Editorial support was provided by PAREXEL. Funding for this study (A39700 and A43288) was provided by Bayer HealthCare Pharmaceuticals, Berlin, Germany.

Conflict of interest F.P., S.L., L.L. and T.S. have no commercial interest, financial interest and/or other relationship with manufacturers of pharmaceuticals, laboratory supplies and/or medical devices or with commercial providers of medically related services. D.H. has cooperation contracts with and has received a grant from Bayer HealthCare Pharmaceuticals. C.S., T.F. and C.G. are full-time employees of Bayer HealthCare Pharmaceuticals.

Open Access This article is distributed under the terms of the Creative Commons Attribution Noncommercial License which permits any noncommercial use, distribution, and reproduction in any medium, provided the original author(s) and source are credited.

\section{References}

1. Guo SW (2009) Recurrence of endometriosis and its control. Hum Reprod Update 15:441-461

2. Batzer FR (2006) GnRH analogs: options for endometriosisassociated pain treatment. J Minim Invasive Gynecol 13:539-545

3. Jee BC, Lee JY, Suh CS, Kim SH, Choi YM, Moon SY (2009) Impact of GnRH agonist treatment on recurrence of ovarian endometriomas after conservative laparoscopic surgery. Fertil Steril 91:40-45

4. Practice Committee of American Society for Reproductive Medicine (2008) Treatment of pelvic pain associated with endometriosis. Fertil Steril 90(5 Suppl):S260-S269

5. Harada T, Momoeda M, Taketani Y, Hoshiai H, Terakawa N (2008) Low-dose oral contraceptive pill for dysmenorrhea associated with endometriosis: a placebo-controlled, double-blind, randomized trial. Fertil Steril 90:1538-1583 
6. Luciano AA (2006) Danazol treatment of endometriosis-associated pain. J Minim Invasive Gynecol 13:523-524

7. Razzi S, Luisi S, Calonaci F, Altomare A, Bocchi C, Petraglia F (2007) Efficacy of vaginal danazol treatment in women with recurrent deeply infiltrating endometriosis. Fertil Steril 88:789-794

8. Vercellini P, Somigliana E, Viganò P, Abbiati A, Barbara G, Crosignani PG (2009) Endometriosis: current therapies and new pharmacological developments. Drugs 69:649-675

9. Rahman M, Berenson AB (2010) Predictors of higher bone mineral density loss and use of depot medroxyprogesterone acetate. Obstet Gynecol 115:35-40

10. Crosignani PG, Olive D, Bergqvist A, Luciano A (2005) Advances in the management of endometriosis: an update for clinicians. Hum Reprod Update 12:179-189

11. Lockhat FB, Emembolu JO, Konje JC (2004) The evaluation of the effectiveness of an intrauterine-administered progestogen (levonorgestrel) in the symptomatic treatment of endometriosis and in the staging of the disease. Hum Reprod 19:179-184

12. Cosson M, Querleu D, Donnez J, Madelenat P, Konincks P, Audebert A, Manhes H (2002) Dienogest is as effective as triptorelin in the treatment of endometriosis after laparoscopic surgery: results of a prospective, multicenter, randomized study. Fertil Steril 77:684-692

13. Köhler G, Faustmann TA, Gerlinger C, Seitz C, Mueck AO (2010) A dose-ranging study to determine the efficacy and safety of 1,2 , and $4 \mathrm{mg}$ of dienogest daily for endometriosis. Int $\mathrm{J}$ Gynaecol Obstet 108:21-25

14. Strowitzki T, Marr J, Gerlinger C, Faustmann T, Seitz C (2010) Dienogest is as effective as leuprolide acetate in treating the painful symptoms of endometriosis: a 24-week, randomized, multicentre, open-label trial. Hum Reprod 25:633-641

15. Momoeda M, Harada T, Terakawa N, Aso T, Fukunaga M, Hagino $\mathrm{H}$, Taketani Y (2009) Long-term use of dienogest for the treatment of endometriosis. J Obstet Gynaecol Res 35:1069-1076
16. Strowitzki T, Faustmann A, Christoph G, Seitz C (2010) Dienogest in the treatment of endometriosis-associated pelvic pain: a 12-week, randomized, double-blind, placebo-controlled study. Eur J Obstet Gynecol Reprod Biol 151:193-198

17. American Society for Reproductive Medicine (1997) Revised American Society for Reproductive Medicine classification of endometriosis: 1996. Fertil Steril 67:817-821

18. Gerlinger C, Endrikat J, Kallischnigg G, Wessel J (2007) Evaluation of menstrual bleeding patterns: a new proposal for a universal guideline based on the analysis of more than 4500 bleeding diaries. Eur J Contracept Reprod Health Care 12:203-211

19. Harada T, Momoeda M, Taketani Y, Aso T, Fukunaga M, Hagino H, Terakawa N (2009) Dienogest is as effective as intranasal buserelin acetate for the relief of pain symptoms associated with endometriosis-a randomized, double-blind, multicenter, controlled trial. Fertil Steril 91:675-681

20. Katsuki Y, Takano Y, Futamura Y, Shibutani Y, Aoki D, Udagawa Y, Nozawa S (1998) Effects of dienogest, a synthetic steroid, on experimental endometriosis in rats. Eur J Endocrinol 138:216-226

21. Okada H, Nakajima T, Yoshimura T, Yasuda K, Kanzaki H (2001) The inhibitory effect of dienogest, a synthetic steroid, on the growth of human endometrial stromal cells in vitro. Mol Hum Reprod 7:341-347

22. Horie P, Harada T, Mitsunari M, Taniguchi F, Iwabe T, Terakawa N (2005) Progesterone and progestational compounds attenuate tumor necrosis factor alpha-induced interleukin-8 production via nuclear factor kappa B inactivation in endometriotic stromal cells. Fertil Steril 83:1530-1535

23. Schindler AE, Henkel A, Moore C, Oettel M (2009) Effect and safety of high-dose dienogest $(20 \mathrm{mg} /$ day $)$ in the treatment of women with endometriosis. Arch Gynecol Obstet 282:507-514 